\title{
Effect of Psychological Status on Outcome of Posterior Lumbar Interbody Fusion Surgery
}

\author{
Raymond Pollock, Sandesh Lakkol, Chakra Budithi, Chandra Bhatia, Manoj Krishna \\ Spinal Unit, University Hospital of North Tees, Stockton-on-Tees, UK
}

Study Design: Prospective longitudinal study.

Purpose: To determine if preoperative psychological status affects outcome in spinal surgery.

Overview of Literature: Low back pain is known to have a psychosomatic component. Increased bodily awareness (somatization) and depressive symptoms are two factors that may affect outcome. It is possible to measure these components using questionnaires.

Methods: Patients who underwent posterior interbody fusion (PLIF) surgery were assessed preoperatively and at follow-up using a self-administered questionnaire. The visual analogue scale (VAS) for back and leg pain severity and the Oswestry Disability Index (ODI) were used as outcome measures. The psychological status of patients was classified into one of four groups using the Distress and Risk Assessment Method (DRAM); normal, at-risk, depressed somatic and distressed depressive.

Results: Preoperative DRAM scores showed 14 had no psychological disturbance (normal), 39 were at-risk, 11 distressed somatic, and 10 distressed depressive. There was no significant difference between the 4 groups in the mean preoperative ODI (analysis of variance, $p=0.426$ ). There was a statistically and clinically significant improvement in the ODI after surgery for all but distressed somatic patients $(9.8$; range, -5.2 to $24.8 ; p=0.177$ ). VAS scores for all groups apart from the distressed somatic showed a statistically and clinically significant improvement. Our results show that preoperative psychological state affects outcome in PLIF surgery.

Conclusions: Patients who were classified as distressed somatic preoperatively had a less favorable outcome compared to other groups. This group of patients may benefit from formal psychological assessment before undergoing PLIF surgery.

Key Words: Spine, Low back pain, Outcomes research, Spinal fusion, Psychological tests

\section{Introduction}

Lumbar pain originates from mechanical and chemical irritation of nociceptors in the lumbar spinal motion segment. However it cannot be thought of as a purely neurophysiological process. As symptom duration exceeds the normal recovery period, psychological and social factors begin to influence the transition to chronicity. It is recognised that psychological and social factors have a role in low back pain [1]. This may explain why spine surgery for low back pain (LBP) in some patients may not produce pain relief despite technically successful surgery and uneventful postoperative recovery.

Patients with LBP appear to be more aware of their bodily sensations [2]. Fear of the condition and that activity will worsen it as well as depressive symptoms are also common

Received Oct 19, 2011; Revised Dec 18, 2011; Accepted Jan 19, 2012

Corresponding author: Raymond Pollock, PhD

Spinal Unit, University Hospital of North Tees,

Hardwick Road, Stockton on Tees, TS19 8PE, UK.

Tel: +44 (0)1642 617617, Fax: +44 (0)1642 383213, E-mail: ray@pollock.org.uk 
in LBP patients. Of the psychological factors, depression has been shown to be predictive of poor outcome [3-6].

There are a number of psychological screening questionnaires commonly used in studies of chronic LBP and spinal surgery outcome. The generic Minnesota Multiphasic Personality Inventory (MMPI) has been widely used and has been shown to enable prediction of poor outcome in spinal surgery [4]. It includes scales for measuring hypochondriases and depression. The Beck Depression Inventory (BDI) [7], Hospital anxiety and depression scale [8] and the modified Zung [9] depression scale are also commonly used instruments for predicting outcome.

There is a lack of questionnaires that measure the level of somatisation. The MMPI contains a scale for hypochondriasis. The health attitude survey [10] was designed to differentiate between somatic and non-somatic patients in primary care. The Modified Somatic Perception Questionnaire (MSPQ) [2] was designed specifically for use with chronic LBP patients. The MSPQ can be combined with the Zung scale to measure psychological distress. This is known as the Distress and Risk Assessment Method (DRAM) [11]. The DRAM was originally developed for predicting outcome of conservative treatment (physiotherapy) in chronic LBP patients.

In this study our aim was to determine the prognostic power of psychological distress when outcome of surgical treatment for LBP is measured.

\section{Materials and Methods}

During the study period March 2004 to November 2008, consecutive patients who met the inclusion-exclusion criteria for posterior interbody fusion (PLIF) surgery were entered into this prospective study at a university hospital in the north east of England. Inclusion criteria were as follows: 1) LBP greater than 2 years duration not responding to conservative therapy including rehabilitation programs, physiotherapy and epidural steroid injections, 2) Oswestry Disability Index (ODI) $>30 ; 3$ ) degenerative disc disease with or without modic changes, 4) spondylolisthesis grade I to III, 5) postlaminectomy/post-discectomy syndrome, and 6) broad based disc prolapse with low back and leg pain. Exclusion criteria were previous spinal infection and spondylolisthesis > grade III.

Patients were assessed preoperatively and at follow-up using a self-administered questionnaire consisting of the MSPQ, Zung, ODI, and visual analogue scale (VAS) for
Table 1. The DRAM decision rules

\begin{tabular}{ll}
\hline \multicolumn{1}{c}{ Classification } & \multicolumn{1}{c}{ Scores } \\
\hline Normal & Zung $<17$ \\
At risk & Zung 17-33, MSPQ $<12$ \\
Distressed depressive & Zung $>33$ \\
Distressed somatic & Zung 17-33, MSPQ $>12$ \\
\hline
\end{tabular}

DRAM: Distress and Risk Assessment Method, MSPQ: Modified Somatic Perception Questionnaire.

back and leg pain.

The MSPQ is a 13 item 4 point self-report questionnaire that measures heightened somatic awareness (somatic anxiety) in chronic back pain patients. The MSPQ has been shown to be a valid and reliable instrument in this patient group. The 13 statements (for example feeling hot all over, nausea, stomach churning) have 4 responses (not at all, little/slightly, a great deal/quite a bit, extremely/could not have been worse) and are scored 0 to 3 respectively, giving a score range of 0 to 39 . The higher the score, the more marked the somatic symptoms.

The Zung depression scale is a 23 item self-report questionnaire that measures depression in LBP patients. Each of the statements (e.g., I feel downhearted and sad, I feel guilty) has 4 choices (rarely or none of the time, some or little of the time, a moderate amount of the time, most of the time), scored either 0 to 3 or 3 to 0 depending on the statement. This results in a score range of 0 to 69 . The higher the score, the more marked the depression.

Psychological status was measured preoperatively using the decision rules of the DRAM (Table 1). This classifies patients into one of 4 types based on a combination of scores from the MSPQ and modified Zung questionnaires: normal (N, Zung score $<17)$ at risk ( $\mathrm{R}$, Zung score 17-33 and MSPQ score $<12$ ), distressed somatic (DS, Zung score 17-33 and MSPQ score $>12$ ) and distressed depressive (DD, Zung score $>33$ ). In the context of spinal surgery, patients who are classified as DD and DS are regarded as those who may have poor outcome after fusion.

The primary outcome measure was the ODI ver. 2.1. A reduction of 16 points on the ODI was considered to be a minimum clinically significant outcome $[12,13]$. Secondary outcomes were the VAS for back and leg pain severity. A decrease pre- to postoperative of 2 was considered as being clinically significant $[12,13]$. The ODI and VAS were measured pre- and postoperatively at 2 years follow-up.

Each patient was seen by a consultant spinal surgeon and 
Table 2. Patient pathology for the series

\begin{tabular}{lc}
\hline \hline \multicolumn{1}{c}{ Diagnosis } & No. $(\%)$ \\
\hline Degenerative disc disease & $54(73)$ \\
Herniated disc & $4(5.4)$ \\
Postlaminectomy/discectomy syndrome & $11(14.9)$ \\
Spondylolisthesis & $5(6.8)$ \\
Total & 74 \\
\hline
\end{tabular}

offered PLIF. All operations were performed by the same consultant surgeon (MK) assisted by a specialist registrar. PLIF is an impatient procedure performed under general anaesthesia. Instrumentation and carbon fibre cages packed with locally harvested bone were used for interbody fusion.

Statistics were obtained using SPSS ver. 14 (SPSS Inc., Chicago, IL, USA). Where the data was skewed, the median in addition to the mean is reported. Before applying parametric methods, the data was checked for normality. If there was significant deviation from normality, non-parametric methods were used. The assumptions of statistical tests were verified before use. Statistical significance was designated at $p<0.05$. The paired samples t-test was used to determine significance between pre- and postoperative outcome scores. All Students' t-tests were two-tailed. Oneway analysis of variance (ANOVA) was used to determine statistical significance between the preoperative outcome scores for the DRAM categories.

\section{Results}

The study group comprised 74 patients (49 females, 25 males; mean age at surgery, 45.6 years; age range, 15 to 70 years). Patients were followed-up for 2 years postoperatively. Symptom duration ranged from 5 to 480 months (mean, 91 months; median, 60 months). Patient pathology is shown in Table 2.

Fifty patients $(67.6 \%)$ were fused at a single level and 24 (32.4\%) had a 2 level fusion. L5/S1 was the most common level fused. From a surgical perspective, fusion was successfully performed on all patients without any serious complications.

The preoperative DRAM scores showed that the 74 patients in the study could be classified into the following types: 14 had no psychological disturbance (normal), 39 were at-risk of developing psychological problems, 11 were DS and 10 DD (Table 3). There was no statistically significant difference in the preoperative ODI (ANOVA, $p=0.426$ ), VAS back (ANOVA, $p=0.36$ ), and VAS leg
(ANOVA, $p=0.059$ ) pain scores for the 4 types of patients.

There was a statistically and clinically significant reduction in the ODI scores after surgery for all but the DS type of patients (Table 3). Similarly the VAS back and leg pain scores for all types apart from the DS showed a statistically and clinically significant improvement (Table 3 ).

\section{Discussion}

The aim of this study was to compare the clinical outcome of patients categorised using the DRAM. We have found that those categorised as DS have the least successful outcome at 24 months as measured by the ODI and VAS back and leg pain scores. Whereas the mean reduction in ODI for the other 3 categories of patients was clinically and statistically significant, this was not the case for the DS patients. They achieved a mean improvement of only 9.8 points, which is less than the clinically significant reduction of 16 points regarded as good outcome [12]. This is in comparison to the normal, at risk and DD with reductions of 20.1, 23.7, and 30.5 respectively. The VAS pain scores also reflect this difference although not as marked as that for the ODI. The reductions in the VAS for all but the DS patients are statistically significant.

Although we have shown that the DRAM is useful as a diagnostic tool for determining those patients who may have poor outcome after spinal surgery we would have expected both distressed categories to have a poor outcome. In fact the depressed patients had a good outcome comparable to the normal and at risk categories. Only the somatisers showed a poor outcome.

The two most important psychological changes that occur in chronic LBP patients are somatisation and depression.

Somatisation is defined here as the experience of psychological distress in the form of physical symptoms. It does not imply that a patient is malingering or is suffering from a psychiatric disorder. Somatisation may be due to the process called psychological sensitization. This may serve to amplify the perception of pain due to idiopathic back problems and transform it into a medical problem. Psychological sensitization may have its basis in neuronal sensitization; an increase in the excitability of neurons so they are more sensitive to stimuli or sensory inputs. A feature of pain sensitization is hypersensitivity in which pain thresholds are lowered; stimuli that would normally not produce pain now do so (allodynia). There is an increase in responsiveness so that stimuli produce an exaggerated and prolonged pain 
(hypoeralgesiua).

What this study shows is that preoperative depression is not a predictor of poor outcome in spinal fusion surgery. This is contrary to the findings of Dvorak et al. [14] and Sørensen and Mors [15] who found that LBP patients with elevated levels of depression as measured by the MMPI had poor outcome after spinal surgery. Studies using other psychometric tests such as the BDI [16] and Zung questionnaire [17] also found that depression predicts poor outcome. We can offer no explanation for this discrepancy when using the DRAM in our study.

In comparing our results with the literature, there has been only a few studies in spinal surgery that have utilised the DRAM as a diagnostic tool for distinguishing patients who may not do well after spinal surgery. Trief et al. [17] found the DRAM to be a significant predictor of outcome in lumbar surgery. Sixty eight percent of procedures were fusion with the remaining being decompressive laminectomy.

In contrast Hobby et al. [18] found the DRAM unable to predict outcome of lumbar discectomy. They found no statistical difference between the DRAM types in postoperative ODI reduction. The explanation for there result may be that the DD and somatic categories of patients were not differentiated; only a combined distressed group was analysed along with the normal and at risk patients.

Similarly, Tandon et al. [19] found the postoperative ODI was unrelated to preoperative psychology as defined using the 4 categories of the DRAM for patients who underwent PLIF surgery. Again, Okoro and Sell [20] used the DRAM to focus on outcome of the somatised category of patients undergoing discectomy or decompression. Using the ODI and VAS leg pain as outcome measures, the authors found no difference between DS and non-somatised patients post surgery.

This study is not without its limitations. This was not a randomised controlled design (level 1 evidence). Our findings that the DRAM is a valid diagnostic tool in identifying patients who may have poor outcome could be tested by conducting a two-arm trial in which patients being considered for surgery are randomised to either receive or not receive presurgical screening using the DRAM. Long-term outcomes of the two arms would be compared after surgical intervention. An alternative study design would use the DRAM to identify somatic patients who would then be subjected to psychological counselling before surgery. 


\section{Conclusions}

In conclusion our results show that in our series, preoperative psychological distress affects outcome of PLIF surgery. Patients who were classified as somatisizers using the DRAM have less favourable outcome than the other categories. This group of patients may benefit from formal psychological assessment before undergoing PLIF surgery. The DRAM appears to be a valid diagnostic test for predicting poor outcome in PLIF surgery.

\section{REFERENCES}

1. Waddell G. Low back pain: a twentieth century health care enigma. Spine (Phila Pa 1976) 1996;21:2820-5.

2. Main CJ. The Modified Somatic Perception Questionnaire (MSPQ). J Psychosom Res 1983;27:503-14.

3. Cashion EL, Lynch WJ. Personality factors and results of lumbar disc surgery. Neurosurgery 1979;4:141-5.

4. Long CJ. The relationship between surgical outcome and MMPI profiles in chronic pain patients. J Clin Psychol 1981;37:744-9.

5. Herron L, Turner JA, Ersek M, Weiner P. Does the Millon Behavioral Health Inventory (MBHI) predict lumbar laminectomy outcome? A comparison with the Minnesota Multiphasic Personality Inventory (MMPI). J Spinal Disord 1992;5:188-92.

6. Pincus T, Burton AK, Vogel S, Field AP. A systematic review of psychological factors as predictors of chronicity/disability in prospective cohorts of low back pain. Spine (Phila Pa 1976) 2002;27:E109-20.

7. Beck AT, Ward CH, Mendelson M, Mock J, Erbaugh J. An inventory for measuring depression. Arch Gen Psychiatry 1961;4:561-71.

8. Zigmond AS, Snaith RP. The hospital anxiety and depression scale. Acta Psychiatr Scand 1983;67:361-70.

9. Zung WW. A Self-Rating Depression Scale. Arch Gen Psychiatry 1965;12:63-70.

10. Noyes R Jr, Langbehn DR, Happel RL, Sieren LR,
Muller BA. Health attitude survey. A scale for assessing somatizing patients. Psychosomatics 1999;40:470-8.

11. Main CJ, Wood PL, Hollis S, Spanswick CC, Waddell G. The distress and risk assessment method. A simple patient classification to identify distress and evaluate the risk of poor outcome. Spine (Phila Pa 1976) 1992;17:42-52.

12. Tafazal SI, Sell PJ. Outcome scores in spinal surgery quantified: excellent, good, fair and poor in terms of patient-completed tools. Eur Spine J 2006;15:1653-60.

13. Hägg O, Fritzell P, Nordwall A; Swedish Lumbar Spine Study Group. The clinical importance of changes in outcome scores after treatment for chronic low back pain. Eur Spine J 2003;12:12-20.

14. Dvorak J, Valach L, Fuhrimann P, Heim E. The outcome of surgery for lumbar disc herniation. II. A 4-17 years' follow-up with emphasis on psychosocial aspects. Spine (Phila Pa 1976) 1988;13:1423-7.

15. Sørensen LV, Mors O. Presentation of a new MMPI scale to predict outcome after first lumbar diskectomy. Pain 1988;34:191-4.

16. Kjellby-Wendt G, Styf JR, Carlsson SG. The predictive value of psychometric analysis in patients treated by extirpation of lumbar intervertebral disc herniation. J Spinal Disord 1999;12:375-9.

17. Trief PM, Grant W, Fredrickson B. A prospective study of psychological predictors of lumbar surgery outcome. Spine (Phila Pa 1976) 2000;25:2616-21.

18. Hobby JL, Lutchman LN, Powell JM, Sharp DJ. The distress and risk assessment method (DRAM). J Bone Joint Surg Br 2001;83:19-21.

19. Tandon V, Campbell F, Ross ER. Posterior lumbar interbody fusion. Association between disability and psychological disturbance in noncompensation patients. Spine (Phila Pa 1976) 1999;24:1833-8.

20. Okoro T, Sell P. The prediction of outcome in somatised patients undergoing elective lumbar surgery. J Bone Joint Surg Br 2009;91:517-21. 